\title{
(2) OPEN ACCESS \\ Long-term outcome of cultivated oral mucosal epithelial transplantation for fornix reconstruction in chronic cicatrising diseases
}

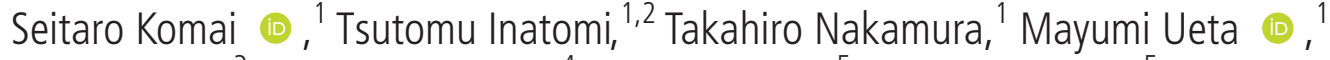 \\ Go Horiguchi, ${ }^{3}$ Satoshi Teramukai, ${ }^{4}$ Yasuko Kimura, ${ }^{5}$ Tatsuo Kagimura, ${ }^{5}$ \\ Masanori Fukushima, ${ }^{6}$ Shigeru Kinoshita ${ }^{0},{ }_{1}^{7}$ Chie Sotozono ${ }^{1}$
}

'Ophthalmology, Kyoto Prefectural University of Medicine, Kyoto 602-8566, Japan

${ }^{2}$ Ophthalmology, National Center for Geriatrics and Gerontology, Obu, Aichi, Japan ${ }^{3}$ Clinical and Translational Research Center, University Hospital, Kyoto Prefectural University of Medicine, Kyoto, Japan

${ }^{4}$ Biostatistics, Kyoto Prefectural University of Medicine, Kyoto, Japan

${ }^{5}$ Translational Research Center for Medical Innovation, Foundation for Biomedical Research and Innovation at Kobe, Kobe, Japan ${ }^{6}$ Medical R\&D, Fukushima \& Partners, Nagoya, Japan ${ }^{7}$ Frontier Medical Science and Technology for Ophthalmology, Kyoto Prefectural University of Medicine, Kyoto, Japan

\section{Correspondence to} Dr Chie Sotozono, Ophthalmology, Kyoto Prefectural University of Medicine, Kyoto, Japan; csotozon@koto.kpu-m.ac.jp

Received 9 December 2020 Revised 7 April 2021 Accepted 17 April 2021
D) Check for updates

(C) Author(s) (or their employer(s)) 2021. Re-use permitted under CC BY-NC. No commercial re-use. See rights and permissions. Published by BMJ.

To cite: Komai S, Inatomi T, Nakamura T, et al.

Br J Ophthalmol Epub ahead of print: [please include Day Month Year]. doi:10.1136/ bjophthalmol-2020-318547

\begin{abstract}
Background/aims To investigate the long-term outcomes of cultivated oral mucosal epithelial transplantation (COMET) for fornix reconstruction in eyes with chronic cicatrising disease.

Methods This retrospective cohort study involved 16 eyes of 15 patients who underwent COMET for symblepharon release and fornix reconstruction between June 2002 and December 2008. The mean postoperative follow-up period was $102.1 \pm 46.0$ months (range: 32-183 months). The treated cicatrising disorders included ocular cicatricial pemphigoid (OCP, five eyes), thermal/chemical injury (three eyes) and other chronic diseases (seven eyes; including recurrent pterygium (two eyes), Stevens-Johnson syndrome (one eye) and graft-versus-host disease (one eye)). Ocular-surface appearance was evaluated before surgery, at 1, 4, 12 and 24 weeks postoperative, and then annually based on the previously reported scoring system. Main outcome measures included overall and disease-specific fornix-reconstruction success probabilities analysed by the Kaplan-Meier survival curve. Symblepharon/fornixshortening recurrence at 24 weeks postoperative, and its relationship to long-term surgical success was also examined.
\end{abstract}

Results At 5 years postoperative, the mean \pm SD overall fornix-reconstruction success probability was $79.6 \% \pm 10.7 \%$, and success probability for thermal/ chemical injury and OCP was $100 \%$ and $53.3 \% \pm 24.8 \%$, respectively ( $p=0.53$, log-rank test). The 3 -year success probability was significantly higher in the no-diseaserecurrence group at 24 weeks postoperative (13 eyes) than in the disease-recurrence group (three eyes) (100\% and $33.3 \% \pm 27.2 \%$, respectively) $(p=0.0073$, log-rank test).

Conclusion COMET was found to be safe and effective for symblepharon release and long-term fornix reconstruction in eyes with chronic cicatrisation. Although the 5-year success probability differed depend on the underlying disease, ocular-surface appearance at 24 weeks postoperative is a factor for predicting longterm outcome.

\section{INTRODUCTION}

Symblepharon and fornix shortening on the ocular surface can result in blink disturbance, and is a condition that is often accompanied by conjunctival invasion and/or loss of vision. These characteristic ocular appearances are commonly observed in patients afflicted with severe ocular-surface diseases (OSDs) such as Stevens-Johnson syndrome (SJS), ocular cicatricial pemphigoid (OCP) and severe thermal/chemical injury. Moreover, recurrent pterygium, tumour resection or repeated eye surgery can initiate symblepharon and end-stage conditions. In eyes with chronic cicatrising diseases, massive inflammation and recurrence of adhesion can easily develop post surgery, thus resulting in a reshortening of the fornix. In such cases, symblepharon release and reconstruction of the fornix conjunctiva are challenging.

It has previously been reported that conjunctiva or mucous membranes (ie, oral and nasal mucosa) have successfully been used for surgical reconstruction of the conjunctiva. ${ }^{1-6}$ Since the report by Tseng et al, ${ }^{7}$ amniotic membrane (AM) transplantation (AMT) has been widely used for conjunctival reconstruction. ${ }^{8-12}$ AMT combined with limbal transplantation or other mucous membrane transplantation techniques for fornix reconstruction have reportedly been successful. ${ }^{12-16}$ Although AMT is the current and widely-accepted surgical technique used for ocular surface reconstruction, its effect is reportedly limited in cases of severe end-stage OSDs, ${ }^{9} 1718$ as the forniceal depth deteriorates over time postsurgery. Hence, and to the best of our knowledge, there are currently no promising treatment methods for achieving longterm surgical success post fornix reconstruction in cases of chronic cicatrising diseases such as severe OSD.

In 2004, Nakamura et $a l^{19}$ reported the successful transplantation of cultivated mucosal epithelial-stem-cell sheets derived from autologous cell sources. Since then, we have performed numerous cultivated oral mucosal epithelial sheet transplantation (COMET) surgeries in patients afflicted with severe OSDs. ${ }^{20-24}$ In our effort to obtain a more deep and broad understanding of the safety and efficacy of COMET, we analysed the clinical data of all 72 patients who underwent COMET since 2002, and our findings revealed that COMET is an extremely effective surgery for reconstruction of the ocular surface and good restoration of vision, ${ }^{25}$ as well as for treating cases of persistent epithelial defect with acute inflammatory activity. ${ }^{26}$ 
The purpose of this present study was to analyse the long-term clinical course in patients who underwent COMET between 2002 and 2008 for fornix reconstruction, and to evaluate the factors for predicting the long-term prognosis.

\section{MATERIALS AND METHODS \\ Patients}

Between June 2002 and December 2008, COMET was performed in 81 eyes of 72 cases with severe OSDs at the Department of Ophthalmology, Kyoto Prefectural University of Medicine, Kyoto, Japan. In this retrospective study, we examined the medical records of all patients who underwent COMET between June 2002 and December 2008. Of those cases, 22 eyes of 21 cases underwent COMET for fornix reconstruction, and of those 21 cases, we retrospectively examined and evaluated the clinical outcome of the cases in which the clinical course could be followed for at least 1-year postoperative.

\section{Cell culture}

All cultivated cell-sheets used for COMET surgery were prepared as previously described, and in accordance with methods approved for Good Manufacturing Practices, as graded by the Cell Processing Centre at Kyoto Prefectural University of Medicine. ${ }^{19}{ }^{27}$ Briefly, autologous oral mucosal epithelial cells were obtained from a 6-mm-diameter biopsy specimen obtained from the buccal mucosa of each patient, and then co-cultured with 3T3 fibroblasts (NIH-3T34; RIKEN Cell Bank, Tsukuba, Japan) inactivated by mitomycin $\mathrm{C}(\mathrm{MMC})$ on $\mathrm{AM}$. The cells were then cultured in medium for 7-10 days, and exposed to air (air-lifting) for 1-2 days. Initially, culture medium containing fetal bovine serum (FBS) was used, yet in later cultures, FBS was replaced with autologous serum in order to reduce the risk of infection by non-human pathogens. ${ }^{27}$ The manufacturing method and the size of the COMET sheets used for fornix reconstruction in this study were identical to those used for corneal reconstruction in our previous reports. ${ }^{25} 26$

\section{Transplantation and postoperative management}

It should be noted that the procedure used for fornix reconstruction slightly differs from that used for corneal reconstruction. In our previous studies of COMET for corneal reconstruction, a single COMET sheet was transplanted to cover the entire cornea. ${ }^{25} 26$ However, in this study, the COMET sheet was divided into two or three pieces, each then being transplanted onto the exposed sclera after release of the symblepharon. In cases with large bare sclera, AM was transplanted onto the sclera, with the COMET sheet then transplanted in combination. In cases with a cataract, cataract surgery was simultaneously performed in combination with COMET. In the cases in which the adhesion was strong, 0.04\% MMC was administered intraoperatively. ${ }^{25} 26$ Postoperative treatment with systemic and local anti-inflammatory medication was performed in the same manner as previously described. ${ }^{25} 26$ Briefly, to prevent postoperative inflammation and immune response, systemic steroids (betamethasone $1 \mathrm{mg} /$ day) and cyclosporine $(2-3 \mathrm{mg}$ / $\mathrm{kg} /$ day) were administered. They were then tapered according to the clinical findings. Dexamethasone $(0.1 \%)$ and antimicrobial eye-drops were instilled four times daily. In cases with dry eye, artificial tears were topically administered. In all cases, a therapeutic soft contact lens was used for at least 1-month postoperative to protect the ocular surface from mechanical damage. 2526 Once stabilisation of the ocular surface was obtained, the anti-inflammatory treatment was continued with a minimal administration of fluorometholone (0.1\%).

\section{Grading system}

Symblepharon score

Symblepharon scores were determined based on the degree of symblepharon, and were defined as ' $0-3$ ' based on the extent of symblepharon formation as follows: score 0: no symblepharon; score 1: symblepharon formation involving only the conjunctival surface; score 2: symblepharon formation on $<50 \%$ of the corneal surface; score 3: symblepharon formation on $>50 \%$ of the corneal surface.

\section{Fornix shortening score}

A fornix shortening score was established, and was graded from ' 0 to 3' based on the clinical features as follows: grade 0: normal depth; grade 1: depth shortened by $<25 \%$; grade 2: depth shortened by $25 \%$ to $50 \%$; grade 3 : depth shortened by $>50 \%$. The upper and lower fornix shortening scores were determined separately, with the fornix shortening score then being defined as the sum of the two scores (maximum Score: 6).

\section{Total adhesion score}

A total adhesion score was established, and was defined as the sum of the symblepharon and fornix-shortening scores (Maximum Score: 9).

\section{Ocular Surface Grading score}

An Ocular Surface Grading (OSG) score was established, with epithelial defects, clinical conjunctivalizsation, neovascularisation, opacification and keratinisation being independently graded based on severity by at least two of three ophthalmologists (CS, TI and SKo) on a scale from ' 0 to 3' in accordance with our previously reported grading system. ${ }^{28}$ The sum of each grading score, including the total adhesion score, was defined as the OSG score (maximum score: 24).

\section{Evaluation of grading scores}

Post-COMET, data were collected from the medical records of the patients. That data included symblepharon, fornix shortening, total adhesion score and OSG score at prior to surgery, at 4, 12 and 24 weeks postoperative, and at 1-year postoperative and each following year thereafter.

\section{Fornix-reconstruction success probability}

Post-COMET, successful fornix reconstruction was defined as the fornix remaining well-reconstructed throughout the postoperative period, and with a shortening score grade of far better than that at prior to surgery. Fornix reconstruction failure was defined as (1) the postoperative total adhesion score becoming equal to, or worse, than that at prior to surgery or (2) any additional fornix-reconstruction surgery being required.

\section{Adverse events}

For the assessment of adverse events related to COMET or the postoperative management, the patients' medical records were examined in regard to proliferative changes, tumour formation, an increase of intraocular pressure (IOP) and the development of infectious keratitis. 


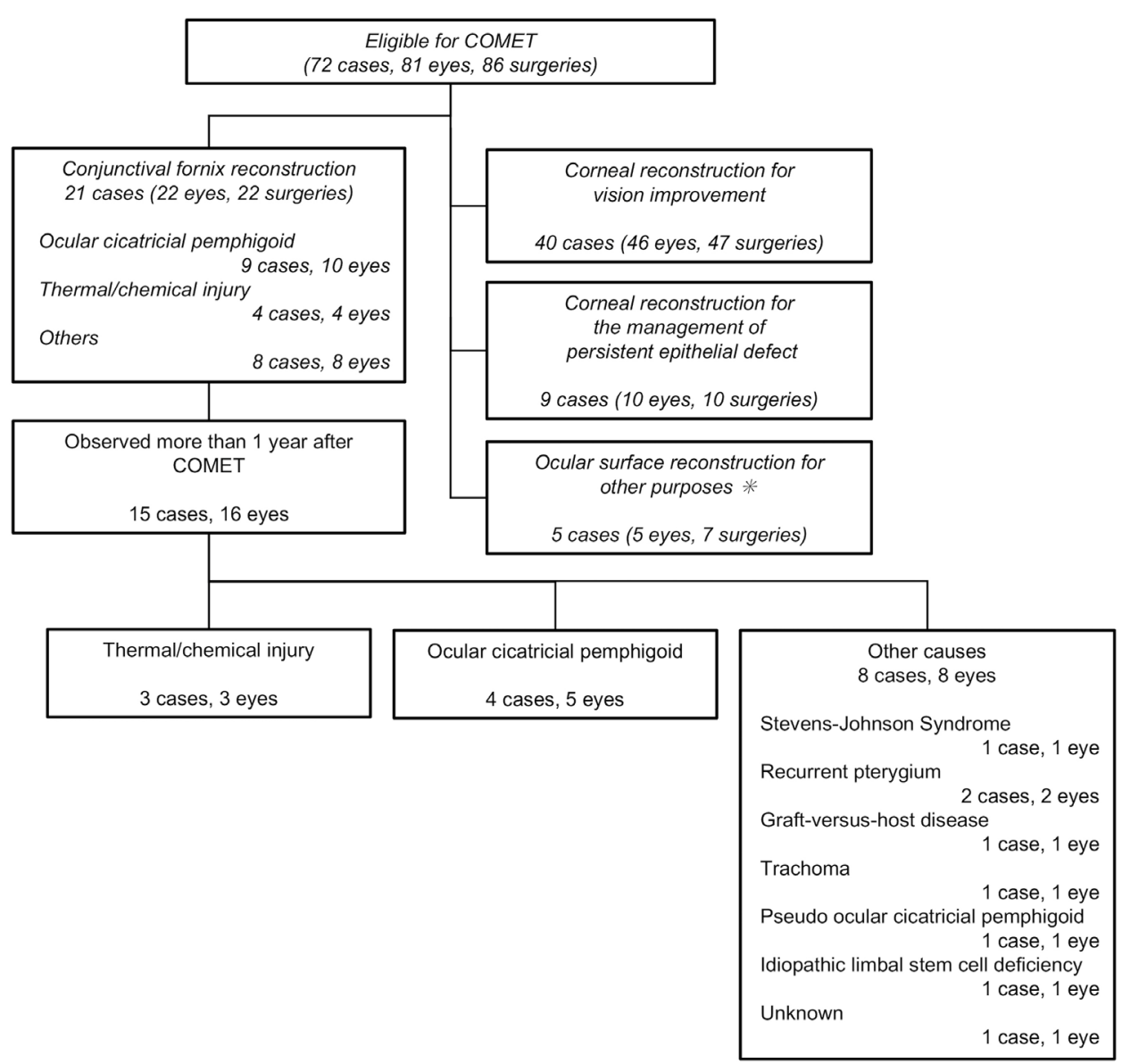

Figure 1 Flow chart diagram of the study. As the data in the flow chart illustrates, 72 patients (81 eyes) underwent cultivated oral mucosal epithelial sheet transplantation (COMET) between June 2002 and December 2008, and 21 patients (22 eyes) underwent COMET for the purpose of fornix reconstruction. The long-term clinical course of fornix reconstruction post-COMET was analysed in 15 patients (16 eyes). Both corneal reconstruction and fornix reconstruction were performed in the same eye in two cases, and counted separately. In one case, bilateral corneal reconstruction and ocular surface reconstruction was performed for cosmetic purposes, and counted as separate cases. * Reconstruction after tumor resection four cases (four eyes, six surgeries) cosmetic one case (one eye, one surgery).

\section{Statistical analysis}

Retrospective cohort study

Recurrence of symblepharon/fornix shortening at 24 weeks postoperative was defined as eyes with at least a ' 1 point' worsening of the symblepharon or fornix shortening score between the 4th and 24th week post-COMET. The Kaplan-Meier method and the log-rank test were used to evaluate the probability of fornix reconstruction being required post-COMET, according to the variables. JMP V.14.0.0 (SAS Institute) statistics software was used for statistical analysis, and a $\mathrm{p}<0.05$ was considered statistically significant.

\section{Short-term pooled analysis}

The changes in the symblepharon score and the fornix shortening score from baseline to at 24 weeks postoperative and the final

\begin{tabular}{|c|c|c|c|c|}
\hline & Thermal/chemical injury & OCP & Other & Total \\
\hline No of eyes & 3 & 5 & 8 & 16 \\
\hline Patient sex (male/female) & $2 / 1$ & $2 / 3$ & $5 / 3$ & $9 / 7$ \\
\hline $\begin{array}{l}\text { Postoperative time-period (months) } \\
\text { Mean } \pm S D\end{array}$ & $72.0 \pm 53.8$ & $95.8 \pm 41.0$ & $117.3 \pm 38.7$ & $102.1 \pm 46.0$ \\
\hline $\begin{array}{l}\text { Fornix shortening score } \\
M e a n \pm S D\end{array}$ & $3.0 \pm 0.8$ & $3.0 \pm 0.6$ & $3.4 \pm 1.4$ & $3.2 \pm 1.1$ \\
\hline $\begin{array}{l}\text { Total adhesion score } \\
\text { Mean } \pm \text { SD }\end{array}$ & $5.3 \pm 0.9$ & $5.6 \pm 1.0$ & $5.5 \pm 2.2$ & $5.5 \pm 1.7$ \\
\hline $\begin{array}{l}\text { Ocular surface grading score } \\
\text { Mean } \pm \text { SD }\end{array}$ & $11.0 \pm 1.6$ & $9.4 \pm 3.0$ & $9.1 \pm 5.2$ & $9.6 \pm 4.2$ \\
\hline
\end{tabular}

COMET, cultivated oral mucosal epithelial sheet transplantation; OCP, ocular cicatricial pemphigoid. 
follow-up examination before the end of 2008 were analysed using the Wilcoxon signed-rank test at the Translational Research Centre for Medical Innovation (Kobe, Japan) using SAS V.9.1 or 8.2 (SAS Institute) statistics software. A p $<0.05$ was considered statistically significant.

\section{RESULTS}

Patients

A flow chart diagram of the study is shown in figure 1 . In this study, 72 patients (81 eyes) underwent COMET between June 2002 and December 2008, and of those 72 patients, 21 (22 eyes) underwent COMET for the purpose of fornix reconstruction. Of the 21 patients ( 22 eyes), 15 patients (16 eyes) were followed for more than 1-year postoperative. The patient characteristics are shown in table 1 . Of those 16 eyes, there were five eyes with OCP, three eyes with thermal or chemical injury and eight eyes with other OSDs (ie, one eye with SJS, two eyes with severe recurrent pterygium, one eye with graft-versus-host disease, one eye with trachoma, one eye with pseudo ocular pemphigoid, one eye with idiopathic stem cell deficiency and one eye with a disease of unknown cause). In those 15 patients, the average (mean \pm SD) age at the time of COMET surgery was $56.8 \pm 17.7$ years (range: 19-83 years) and the average postoperative follow-up period was $102.1 \pm 46.0$ months (range: $32-183$ months). At the time of undergoing COMET surgery, all cases were in the chronic phase with slight or no conjunctivitis and low disease activity (figure 2). Of the 16 treated eyes, fornix reconstruction was well maintained in 12 eyes and eventually failed in 4 eyes during the postoperative follow-up period (figure 2) (table 2). In the two cases that failed, additional surgery for recurrent adhesion was performed; that is, AMT was performed in the one case with OCP (case 5), and keratoepithelioplasty with AMT was performed in the one case with recurrent pterygium (case 10).

\section{Fornix-reconstruction success probability}

The overall average probability of successful fornix-reconstruction at 5 and 10 years postoperative was $79.6 \% \pm 10.7 \%$ and $70.7 \% \pm 12.6 \%$, respectively. Fornix reconstruction failed in two patients with OCP, one patient with recurrent pterygium and one patient with trachoma. The disease-specific probability of successful fornix-reconstruction was also analysed. The mean probability of surgical success at 5 and 10 years postoperative for the patients with thermal/chemical injury, OCP and 'other disease', respectively, was $100 \%, 53.3 \% \pm 24.8 \%$, and $87.5 \% \pm 11.7 \%$, and $100 \%$, $53.3 \% \pm 24.8 \%$, and $72.9 \% \pm 16.5 \%$ (figure 3 ).

\section{Association between patient preoperative background and fornix-reconstruction success probability}

Although univariate analysis demonstrated no significant differences in the fornix-reconstruction success probability between three groups divided by their specific underlying disease, the mean probability at 10 years post-COMET was found to be the poorest in the OCP patients (ie, $53.3 \% \pm 24.8 \%$ in the OCP patients compared with $100 \%$ in the patients with thermal/chemical injury and $72.9 \% \pm 16.5 \%$ in the patients with other diseases $(p=0.53, \log$-rank test)).

\section{Association between fornix-reconstruction success probability and postoperative surgical outcome}

We also examined the relationship between the fornix-reconstruction success probability and surgical outcomes at 24 weeks postoperative. In relation to the presence of the recurrence of symblepharon/fornix shortening at 24 weeks postoperative, the 16 treated eyes were

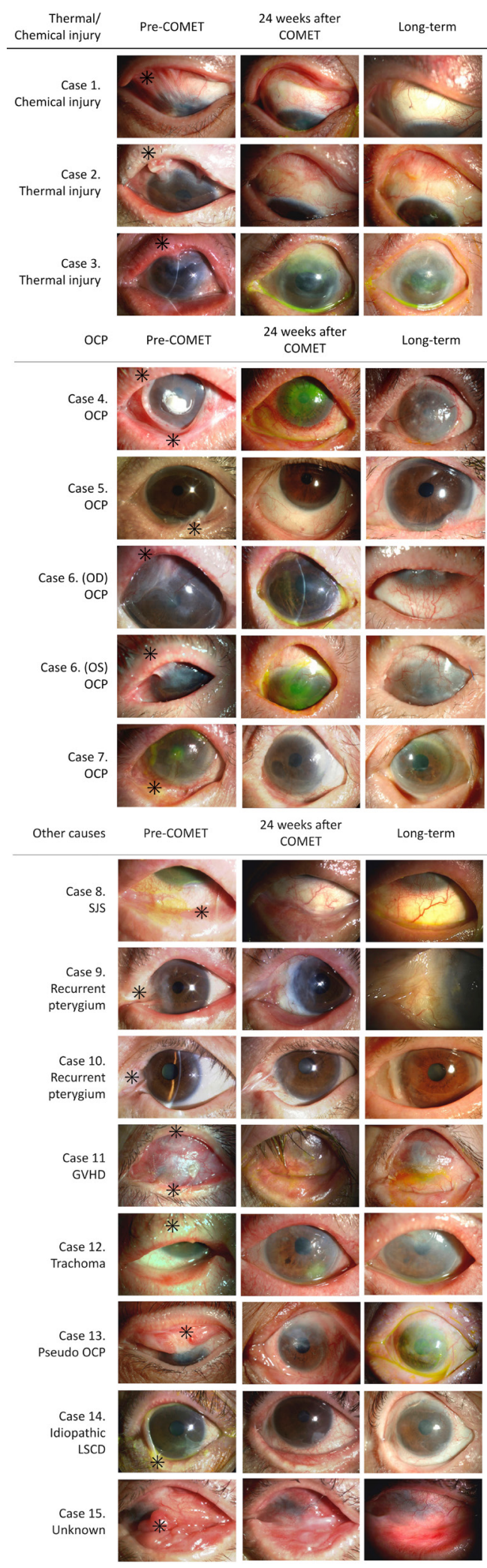

Figure 2 Slit-lamp images obtained at prior to surgery and at 24 weeks and long-term postoperative. Slit-lamp images obtained at prior to surgery and at 24 weeks and long-term postoperative of 16 eyes that underwent cultivated oral mucosal epithelial sheet transplantation (COMET) for fornix reconstruction are showed. *Shows the area of COMET. COMET, cultivated oral mucosal epithelial sheet transplantation; GVHD, Graft-versus-host disease; LSCD, Limbal stem cell deficiency; $0 C P$, ocular cicatricial pemphigoid; SJS, Stevens-Johnson syndrome. 
Table 2 Change in total adhesion score over time

\begin{tabular}{|c|c|c|c|c|c|c|c|c|c|c|c|c|c|c|}
\hline \multirow[b]{2}{*}{ Case } & \multirow[b]{2}{*}{ Age/sex } & \multirow[b]{2}{*}{ Primary Disease } & \multirow{2}{*}{$\begin{array}{l}\text { Duration from } \\
\text { The onset (years) }\end{array}$} & \multirow{2}{*}{$\begin{array}{l}\text { Follow-up } \\
\text { Period (years) }\end{array}$} & \multicolumn{9}{|c|}{ Total adhesion score } & \multirow{2}{*}{$\begin{array}{l}\text { Time to fail } \\
\text { (years) }\end{array}$} \\
\hline & & & & & Pre & 4 weeks & 24 weeks & 1 year & 2 years & 3 years & 5 years & 10 years & Result & \\
\hline \multicolumn{15}{|c|}{ A. Thermal/chemical injury } \\
\hline 1 & $35-39 / \mathrm{M}$ & Chemical injury & 9.9 & 12.3 & 6 & 0 & 0 & 2 & 2 & 2 & 2 & 2 & $S$ & - \\
\hline 2 & $30-34 / \mathrm{M}$ & Thermal injury & 1.3 & 3 & 4 & 0 & 0 & 1 & 1 & 1 & - & - & $S$ & - \\
\hline 3 & $70-74 / F$ & Thermal injury & unknown & 2.7 & 6 & 0 & 0 & 0 & 0 & 0 & - & - & $S$ & - \\
\hline \multicolumn{15}{|c|}{ B. Ocular cicatricial pemphigoid } \\
\hline 4 & $65-69 / \mathrm{M}$ & OCP & unknown & 12.8 & 4 & 0 & 0 & 0 & 0 & 0 & 0 & 0 & $S$ & - \\
\hline 5 & $55-59 / \mathrm{M}$ & OCP & 4.6 & 10.2 & 5 & 0 & 0 & 2 & 2 & 4 & $(5)^{*}$ & - & $\mathrm{F}$ & 3.7 \\
\hline $6(O D)$ & $70-74 / F$ & OCP & unknown & 8.4 & 7 & 0 & 3 & 5 & 5 & $(7)^{*}$ & 7 & - & $\mathrm{F}$ & 2.5 \\
\hline (OS) & $75-80 / F$ & OCP & unknown & 5.6 & 6 & 1 & 1 & 3 & 3 & 3 & 3 & - & $S$ & - \\
\hline 7 & $80-84 / \mathrm{F}$ & OCP & 10.2 & 3 & 6 & 0 & 0 & 3 & 4 & 4 & - & - & $S$ & - \\
\hline \multicolumn{15}{|c|}{ C. Other } \\
\hline 8 & $50-54 / \mathrm{M}$ & SJS & 0.5 & 7.3 & 3 & 0 & 0 & 1 & 1 & 1 & 1 & - & $S$ & - \\
\hline 9 & $55-59 / \mathrm{M}$ & Recurrent pterygium & 20 & 12.5 & 4 & 0 & 0 & 2 & - & - & 2 & 2 & $S$ & - \\
\hline 10 & $65-69 / F$ & Recurrent pterygium & 11 & 9.7 & 2 & 0 & $(4)^{*}$ & - & - & - & - & - & $\mathrm{F}$ & 0.5 \\
\hline 11 & 15-19/F & GVHD & 6.8 & 5.3 & 7 & 0 & 3 & 3 & - & 3 & 3 & - & $S$ & - \\
\hline 12 & $70-74 / F$ & Trachoma & 60 & 5.8 & 7 & 0 & 0 & 0 & 0 & 0 & 0 & $(5)^{\star}$ & $\mathrm{F}$ & 5.6 \\
\hline 13 & 55-59/M & Pseudo OCP & 4 & 10.4 & 7 & 0 & 0 & 1 & 1 & 1 & 1 & 1 & $S$ & - \\
\hline 14 & $55-59 / \mathrm{M}$ & Idiopathic LSCD & 3 & 11.8 & 5 & 0 & 0 & 2 & - & - & - & 2 & $S$ & - \\
\hline 15 & $30-34 / \mathrm{M}$ & Unknown & 0.3 & 15.3 & 9 & 6 & 6 & 8 & - & - & 8 & 8 & $S$ & - \\
\hline
\end{tabular}

*Shows the total adhesion score at the time of failure of fornix reconstruction.

GVHD, graft-versus-host disease; LSCD, limbal stem cell deficiency; OCP, ocular cicatricial pemphigoid; SJS, Stevens-Johnson syndrome.

divided into the following two groups: (1) the eyes in which recurrence occurred (recurrent group, 3 eyes) and (2) the eyes in which recurrence did not occur (non-recurrent group, 13 eyes). At 3 years postoperative, the fornix-reconstruction probability in the recurrent group was $33.3 \% \pm 27.2 \%$, while that of non-recurrence group was $100 \%$ (figure 4). Univariate analysis showed a significant difference for recurrence $(p=0.0073, \log$-rank test).

\section{Disease-specific differences during 24-weeks post-COMET}

The disease-specific symblepharon scores and fornix shortening scores at prior to surgery and at 4, 12 and 24 weeks postoperative are shown in figure 5. In cases with thermal/chemical injury, both symblepharon and fornix shortening scores improved throughout 24 weeks postoperative in three out of four eyes (75\%) (95\% CI 19.4 to 99.4) while one patient failed to undergo the 24-week-postoperative follow-up examination.

In cases with OCP, improvement of the fornix shortening score was maintained at 24 weeks postoperative in 7 out of 10 eyes $(70 \%)(\mathrm{p}=0.0313$, Wilcoxon signed-rank test, 95\% CI 34.8 to 93.3 ), and improvement of the symblepharon score was maintained in 6 out of 10 eyes $(60 \%)(p=0.0625$, Wilcoxon

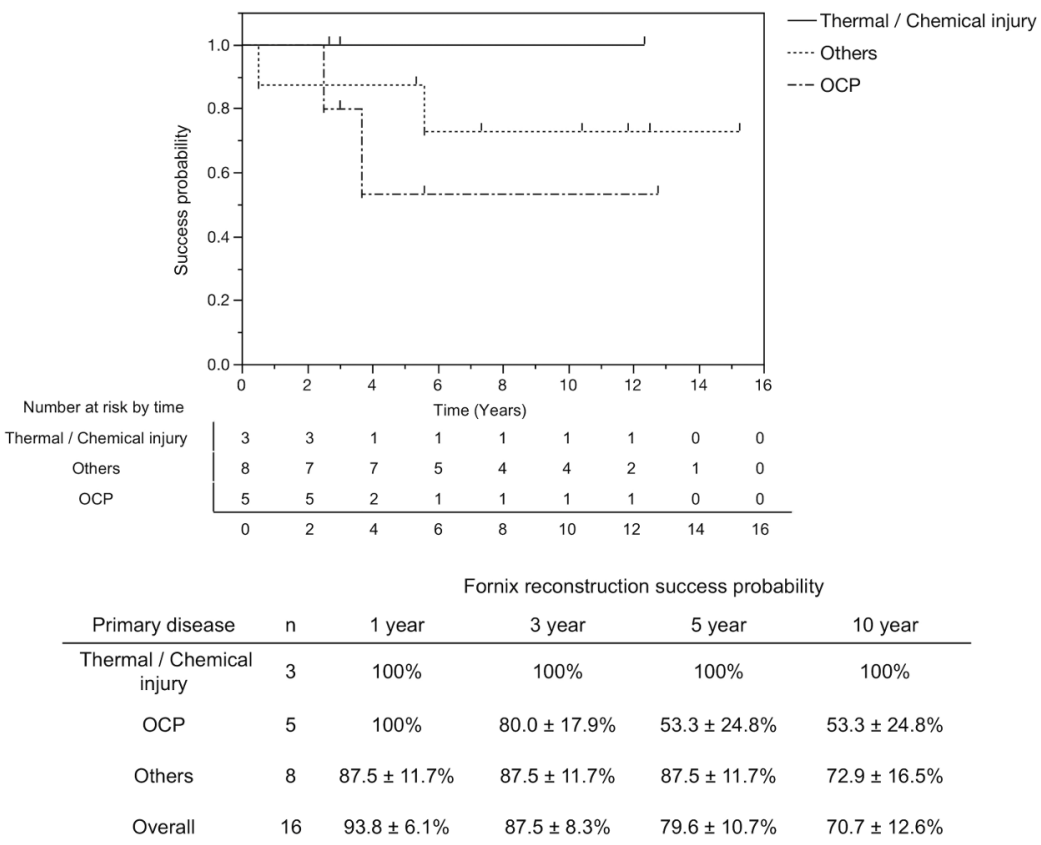

Figure 3 Fornix-reconstruction success probability assessed by Kaplan-Meier survival curves: comparison of 3 groups divided according to the preoperative diagnosis. Diagram showing the fornix-reconstruction success probability assessed by Kaplan-Meier survival curves. There was no significant difference in success probability among the ocular cicatricial pemphigoid (OCP), thermal/chemical injury, and 'other' groups ( $p=0.5297$, log-rank test). 


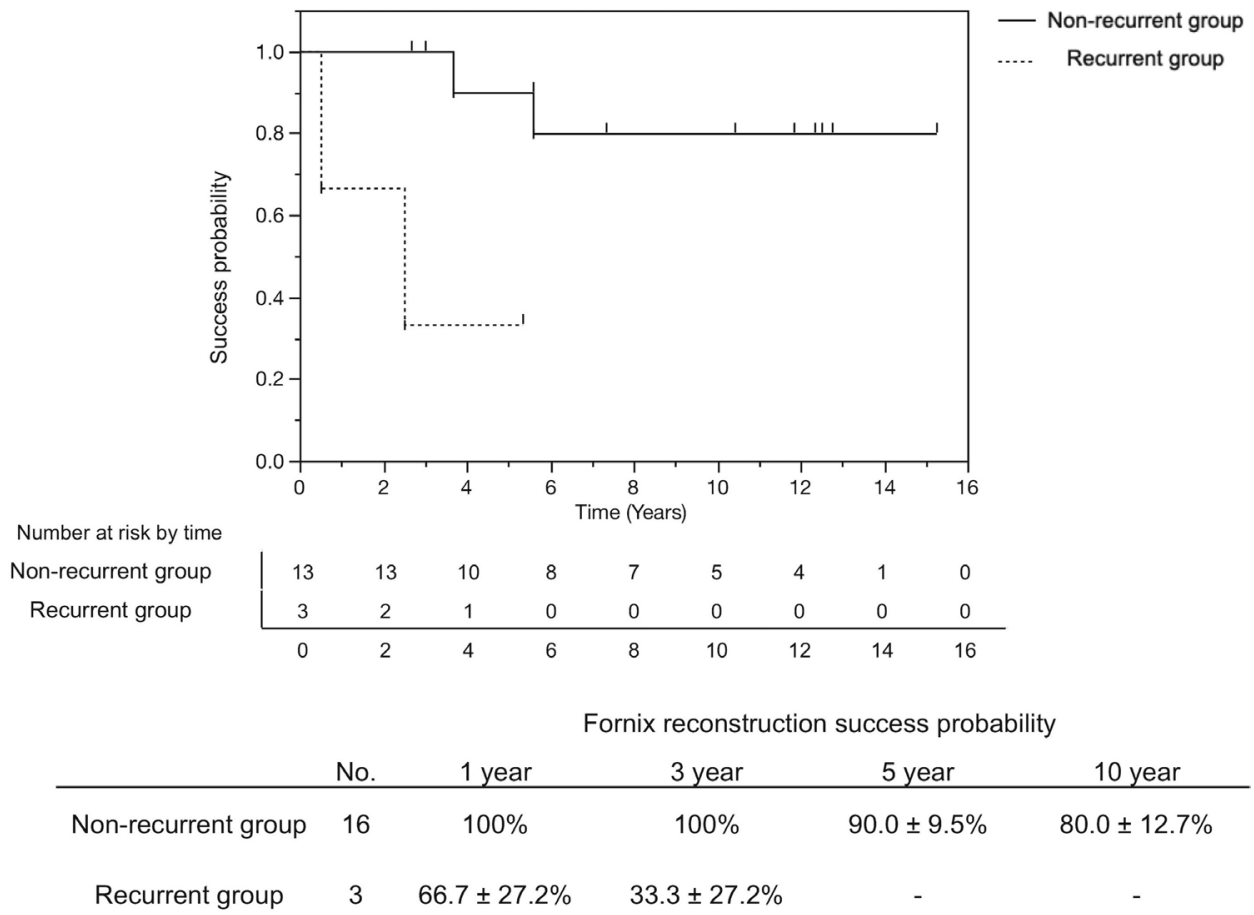

Figure 4 Fornix-reconstruction success probability assessed by Kaplan-Meier survival curves: comparison of two groups with or without recurrence of adhesion at 24 weeks post-COMET. Diagram showing the fornix-reconstruction success probability assessed by Kaplan-Meier survival curves. To examine the presence of the recurrence of symblepharon/fornix shortening at 24 weeks postoperative, the 16 treated eyes were divided into the following two groups: (1) the eyes in which recurrence occurred (recurrent group, 3 eyes) and (2) the eyes in which recurrence did not occur (nonrecurrent group, 13 eyes). At 3 years postoperative, the fornix-reconstruction probability in the recurrent group was $33.3 \% \pm 27.2 \%$, while that of non-recurrent group was $100 \%$. Univariate analysis showed a significant difference for recurrence ( $p=0.0073$, log-rank test). COMET, cultivated oral mucosal epithelial sheet transplantation.

signed-rank test, $95 \%$ CI 26.2 to 87.8 ). However, 3 of the 10 eyes failed to undergo the requested follow-up examination at 24 weeks postoperative. It should be noted that until 24 weeks postoperative, an increase in both scores was observed in one eye with OCP.

\section{Adverse events}

Our findings revealed that there was no proliferative change of the transplanted COMET sheet and no tumour formation in the 16 treated eyes, which is important. In 1 (6.3\%) of the 16 treated eyes, conjunctival epithelial defect was observed at 63 months post-COMET, and recurrent adhesion was found to have developed at the site of the defect.

During the postoperative follow-up period, an increase of IOP was observed in four eyes (25\%), yet in three of those four eyes, the IOP increase was controlled via the administration of antiglaucoma eye drops. In the remaining one eye, trabeculotomy was performed at 61 months post-COMET. Although traumaassociated corneal infection occurred in one eye, there was no development of infectious keratitis related to the COMET surgery in that eye.

\section{DISCUSSION}

The findings in this study demonstrate the long-term safety and efficacy of COMET for fornix reconstruction in chronic cicatrising diseases. The mean overall fornix-reconstruction success probability at 5 and 10 years postoperative was $79.6 \% \pm 10.7 \%$ and $70.7 \% \pm 12.6 \%$, respectively. In all patients, no proliferative changes, such as ocular-surface squamous cell carcinoma, developed post-COMET. In this current study, we analysed the clinical courses post-COMET for up to 153 months (average: $102.1 \pm 46.0$ months), and to the best of our knowledge, this is the first report on the long-term surgical outcomes of regenerative medicine over a nearly 10 -year postoperative period, on average.

Interestingly, our findings revealed that the long-term outcome of COMET for fornix reconstruction was influenced by the underlying disease. The postoperative course in the patients with thermal or chemical injury was very stable, and there was no recurrence of adhesion in all cases. On the other hand, the patients with OCP had a clearly poorer prognosis than the patients with thermal or chemical injury, although statistical analysis showed no significant difference, probably due to the small number of patients.

In regard to the ocular surface findings at the early period post COMET, the group in which fornix adhesions recurred until 24 weeks post-COMET had a poorer prognosis than the group with no recurrence. In our previous analysis of the longterm results of COMET for corneal-surface reconstruction, the activity of superficial corneal vascularisation was found to have decreased until 6 months post-COMET. ${ }^{24}$ It is generally thought that the ocular surface becomes stabilised at 6 months post-COMET. Thus, in cases that undergo COMET for fornix reconstruction, strict and careful management is vital when an exacerbation of adhesion occurs during the 6-month postoperative period.

It has been previously reported that the presence of postoperative chronic inflammation or underlying autoimmune diseases such as OCP may contribute to a decreased surgical outcome post ocular surface reconstruction in cases of severe OSD. ${ }^{8929-31}$ 


\section{A. Thermal/Chemical injury}
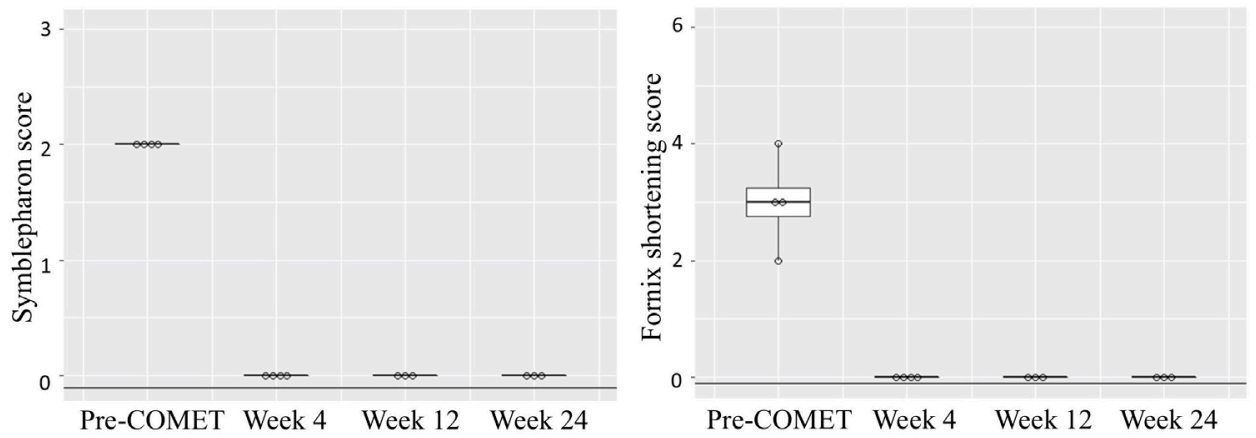

\section{B. Ocular cicatricial pemphigoid}
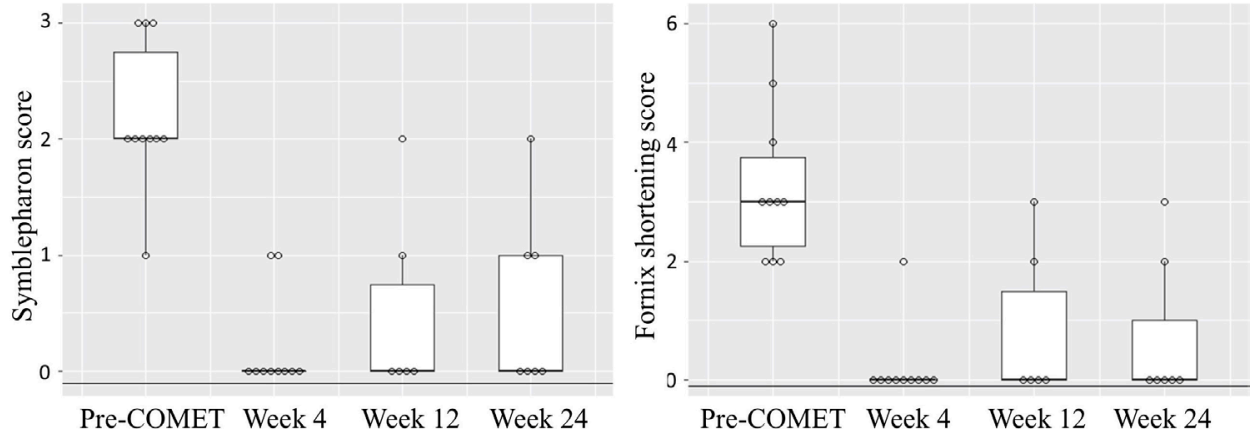

Figure 5 Box-plot graphs showing the preoperative and postoperative clinical outcomes of COMET at 24 weeks postoperative. Box-plot graphs showing the preoperative data and the clinical outcomes at 24 weeks postoperative of 4 eyes of thermal/chemical injury and 10 eyes of OCP that underwent COMET symblepharon and fornix reconstruction. (A) Thermal/chemical injury group ( $\mathrm{n}=4$ eyes). Both symblepharon and fornix shortening scores were improved in all 4 cases at 4, 12 and 24 weeks postoperative compared with at prior to surgery. No progression of symblepharon or fornix shortening is observed in all eyes until 24 weeks postoperative. (B) OCP group ( $n=10$ eyes). The symblepharon score improved significantly ( $p=0.0313$, Wilcoxon signed-rank test) at 4 weeks postoperative. The significant improvement of the fornix shortening scores was maintained at each period ( $p=0.0313$ at each period). COMET, cultivated oral mucosal epithelial transplantation; OCP, ocular cicatricial pemphigoid.

In this present study, postoperative failure of the transplanted graft occurred in two eyes with OCP (cases 5 and 6), yet in one of those two eyes (case 5), good visual acuity (20/20) was found to be well maintained at 122 months postoperative, despite twice performing additional surgery for the release of the adhesion. Thus, we consider that case to be a clinical success, since surgical intervention in that eye resulted in good visual acuity being maintained for over 10 years postoperative.

It should be noted that the present study did have some limitations. First, this study involved only a small number of patients. Second, there was no control group in this study. Thus, it might be preferable to conduct large-scale prospective clinical study in the future in order to compare COMET and AMT for fornix reconstruction.

In conclusion, the findings of this retrospective study revealed that COMET for fornix reconstruction is a safe and effective method to treat symblepharon and fornix shortening over a long-term period, and also suggest that the postoperative course until 24 weeks postoperative may be a predictive factor of the long-term success of fornix reconstruction surgery. Since the primary disease is also considered to be associated with the long-term prognosis, control of that disease with appropriate management may lead to the clinical success of COMET for fornix reconstruction.

Acknowledgements The authors wish to thank John Bush for reviewing the manuscript.
Contributors Drafting of the manuscript: SKo, TI, ST, SKi and CS. Study concept and design: TI, TN, MF, SKi and CS. Analysis and interpretation: SKo, TI, YK, MF and CS. Provision of materials, patients, or resources: SKo, TI, TN, MU, SKi and CS. Statistical expertise: GH, ST and TK. All authors provided significant effort in the discussion of the results, reviewing the manuscript and final approval of the manuscript.

Funding This study was supported by a Grant-in-aid for Scientific Research from the Japanese Ministry of Health, Labor and Welfare (19H03809), a Research Grant from the Japan Agency for Medical Research and Development (19lm0203013h) and a Research Grant from the Japanese Ministry of Education, Culture, Sports, Science and Technology (20FC103).

Disclaimer The author(s) make the following disclosures: CS: grants from Japan Agency for Medical Research and Development (AMED), grants from Ministry of Health, Labour and Welfare, Japan, grants from Japanese Ministry of Education, Culture, Sports, Science and Technology, during the conduct of the study. SKi: grants from Japan Agency for Medical Research and Development (AMED), grants from Ministry of Health, Labour and Welfare, Japan, grants and personal fees from Santen Pharmaceutical, grants and personal fees from Otsuka Pharmaceutical, grants and personal fees from Senju Pharmaceutical, grants and personal fees from Kowa, grants from HOYA, grants from Oncolys Biopharma, grants from LION, grants from CorneaGen, personal fees from Alcon Japan, personal fees from Abbott Medical Optics, personal fees from Novartis, personal fees from Astellas Pharma, outside the submitted work.

Competing interests None declared.

Patient consent for publication Not required.

Ethics approval Approval of the study protocol was obtained from the Institutional Review Board of Kyoto Prefectural University of Medicine, Kyoto, Japan in 2002, and in accordance with the tenets set forth in the Declaration of Helsinki for research involving human subjects, written informed consent was obtained from all patients prior to surgery. Moreover, approval was obtained from the Ethical Review Board of Kyoto Prefectural University of Medicine for the mid- 
term meta-analysis in 2009 and for this present long-term retrospective cohort study in 2017.

Provenance and peer review Not commissioned; externally peer reviewed.

Data availability statement All data relevant to the study are included in the article or uploaded as online supplemental information. The data are deidentified participant data. The data are not available.

Open access This is an open access article distributed in accordance with the Creative Commons Attribution Non Commercial (CC BY-NC 4.0) license, which permits others to distribute, remix, adapt, build upon this work non-commercially, and license their derivative works on different terms, provided the original work is properly cited, appropriate credit is given, any changes made indicated, and the use is non-commercial. See: http://creativecommons.org/licenses/by-nc/4.0/.

ORCID iDs

Seitaro Komai http://orcid.org/0000-0002-4208-8966

Mayumi Ueta http://orcid.org/0000-0002-2678-5024

Shigeru Kinoshita http://orcid.org/0000-0002-6839-251X

\section{REFERENCES}

1 Hosni FA. Repair of trachomatous cicatricial entropion using mucous membrane graft. Arch Ophthalmol 1974;91:49-51.

2 Meighan TS. On an operation for symblepharon by transplantation of mucous membrane from the lip. Br Med J 1889;1:706-7.

3 Vastine DW, Stewart WB, Schwab IR. Reconstruction of the periocular mucous membrane by autologous conjunctival transplantation. Ophthalmology 1982;89:1072-81.

4 Naumann GO, Lang GK, Rummelt V, et al. Autologous nasal mucosa transplantation in severe bilateral conjunctival mucus deficiency syndrome. Ophthalmology 1990;97:1011-7.

5 Shore JW, Foster CS, Westfall CT, et al. Results of buccal mucosal grafting for patients with medically controlled ocular Cicatricial pemphigoid. Ophthalmology 1992;99:383-95.

6 Kuckelkorn R, Schrage N, Redbrake C, et al. Autologous transplantation of nasal mucosa after severe chemical and thermal eye burns. Acta Ophthalmol Scand 1996;74:442-8.

7 Kim JC, Tseng SC. Transplantation of preserved human amniotic membrane for surface reconstruction in severely damaged rabbit corneas. Cornea 1995;14:473???484-84.

8 Solomon A, Espana EM, Tseng SCG. Amniotic membrane transplantation for reconstruction of the conjunctival fornices. Ophthalmology 2003;110:93-100

9 Barabino S, Rolando M, Bentivoglio G, et al. Role of amniotic membrane transplantation for conjunctival reconstruction in ocular-cicatricial pemphigoid. Ophthalmology 2003;110:474-80.

10 Honavar SG, Bansal AK, Sangwan VS, et al. Amniotic membrane transplantation for ocular surface reconstruction in Stevens-Johnson syndrome. Ophthalmology 2000;107:975-9.

11 Tseng SC, Prabhasawat P, Lee SH. Amniotic membrane transplantation for conjunctival surface reconstruction. Am J Ophthalmol 1997;124:765-74.

12 Tseng SCG, Di Pascuale MA, Liu DT-S, et al. Intraoperative mitomycin C and amniotic membrane transplantation for fornix reconstruction in severe Cicatricial ocular surface diseases. Ophthalmology 2005;112:896-903.
13 Jain S, Rastogi A. Evaluation of the outcome of amniotic membrane transplantation for ocular surface reconstruction in symblepharon. Eye 2004;18:1251-7.

$14 \mathrm{Kim} \mathrm{JH}$, Chun YS, Lee SH, et al. Ocular surface reconstruction with autologous nasal mucosa in Cicatricial ocular surface disease. Am J Ophthalmol 2010;149:45-53.

15 Liu J, Sheha H, Fu Y, et al. Oral mucosal graft with amniotic membrane transplantation for total limbal stem cell deficiency. Am J Ophthalmol 2011;152:739-47

16 Kheirkhah A, Ghaffari R, Kaghazkanani R, et al. A combined approach of amniotic membrane and oral mucosa transplantation for fornix reconstruction in severe symblepharon. Cornea 2013;32:155-60.

17 Rahman I, Said DG, Maharajan VS, et al. Amniotic membrane in ophthalmology: indications and limitations. Eye 2009;23:1954-61.

18 Walkden A. Amniotic membrane transplantation in ophthalmology: an updated perspective. Clin Ophthalmol 2020;14:2057-72.

19 Nakamura T, Inatomi T, Sotozono C, et al. Transplantation of cultivated autologous oral mucosal epithelial cells in patients with severe ocular surface disorders. $\mathrm{Br} J$ Ophthalmol 2004;88:1280-4.

20 Nishida K, Yamato M, Hayashida Y, et al. Corneal reconstruction with tissueengineered cell sheets composed of autologous oral mucosal epithelium. $N$ Engl J Med 2004;351:1187-96.

21 Inatomi T, Nakamura T, Koizumi N, et al. Midterm results on ocular surface reconstruction using cultivated autologous oral mucosal epithelial transplantation. Am J Ophthalmol 2006:141:267-75.

22 Chen H-CJ, Chen H-L, Lai J-Y, et al. Persistence of transplanted oral mucosal epithelial cells in human cornea. Invest Ophthalmol Vis Sci 2009:50:4660-8.

23 Takeda K, Nakamura T, Inatomi T, et al. Ocular surface reconstruction using the combination of autologous cultivated oral mucosal epithelial transplantation and eyelid surgery for severe ocular surface disease. Am J Ophthalmol 2011:152:195-201.

24 Nakamura T, Takeda K, Inatomi T, et al. Long-Term results of autologous cultivated oral mucosal epithelial transplantation in the scar phase of severe ocular surface disorders. Br J Ophthalmol 2011;95:942-6.

25 Sotozono C, Inatomi T, Nakamura T, et al. Visual improvement after cultivated ora mucosal epithelial transplantation. Ophthalmology 2013;120:193-200.

26 Sotozono C, Inatomi T, Nakamura T, et al. Cultivated oral mucosal epithelial transplantation for persistent epithelial defect in severe ocular surface diseases with acute inflammatory activity. Acta Ophthalmol 2014:92:e447-53.

27 Ang LPK, Nakamura T, Inatomi T, et al. Autologous serum-derived cultivated oral epithelial transplants for severe ocular surface disease. Arch Ophthalmol 2006;124:1543-51.

28 Sotozono C, Ang LPK, Koizumi N, et al. New grading system for the evaluation of chronic ocular manifestations in patients with Stevens-Johnson syndrome. Ophthalmology 2007;114:1294-302.

29 Ilari L, Daya SM. Long-Term outcomes of keratolimbal allograft for the treatment of severe ocular surface disorders. Ophthalmology 2002:109:1278-84.

30 Solomon A, Ellies P, Anderson DF, et al. Long-Term outcome of keratolimbal allograft with or without penetrating keratoplasty for total limbal stem cell deficiency. Ophthalmology 2002;109:1159-66.

31 Foster CS, Sainz De La Maza M. Ocular Cicatricial pemphigoid review. Curr Opin Allergy Clin Immunol 2004;4:435-9. 